MRS Proceedings 1712, 2014, Article 732

DOI: $10.1557 /$ opl.2014.732

Version as-accepted by publisher.

\title{
In-situ annealing of self-ion irradiation damage in tungsten
}

Xiaoou. $\mathrm{Yi}^{1}$, Michael L. Jenkins ${ }^{2}$, Steve G. Roberts ${ }^{1}$ and Marquis A. Kirk ${ }^{3}$

${ }^{1}$ Department of Materials, University of Oxford, Parks Road, Oxford, OX1 3PH, U.K.

${ }^{2}$ Trinity College, University of Oxford, Broad Street, Oxford, OX1 3BH, U.K.

${ }^{3}$ Materials Science Division, Argonne National Laboratory, Argonne, IL 60439, U.S.A.

\begin{abstract}
In our earlier work [1] microstructural evolution in tungsten under self-ion irradiation was investigated as a function of temperature and dose by in-situ $150 \mathrm{keV} \mathrm{W}^{+}$ion irradiations on the IVEM-Tandem facility at Argonne National Laboratory (ANL). The present work focuses on the thermal stability of this damage. Thin foils of tungsten were irradiated at room temperature (R.T.) to fluences up to $10^{18} \mathrm{~W}^{+} \mathrm{m}^{-2}(\sim 1.0 \mathrm{dpa})$ and were then annealed in-situ for up to $120 \mathrm{~min}$ at temperatures between 300 and $800^{\circ} \mathrm{C}$.

We found that: (1) loops with Burgers vectors $1 / 2<111\rangle$ and $<100\rangle$ coexist during annealing; (2) $<100\rangle$ is not a stable loop configuration above $300^{\circ} \mathrm{C}$ and the fraction of such loops decreased with increasing temperature and/or time; (3) changes in loop populations during annealing were very sensitive to temperature, but less sensitive to time. The majority of changes occurred within $15 \mathrm{~min}$, and were associated with the loss of small (1-2 nm) dislocation loops. The origin of these trends is discussed by considering defect mobility and the energetics of defect configurations predicted by previous DFT calculations [2].
\end{abstract}

\section{INTRODUCTION}

Tungsten is a prime candidate for building divertor components in fusion reactors. During service, this component would be subject to fast neutron displacement damage at temperatures between $\sim 500^{\circ} \mathrm{C}-1200^{\circ} \mathrm{C}$, depending on location. Six annealing stages of radiation-induced damage in neutron irradiated tungsten have been identified based on the recovery of electrical conductivity during heat treatments [3-6]. Each corresponds to the migration of one or a few defect species. The stages relevant to fusion power applications are: stage III $\left(130-450^{\circ} \mathrm{C}\right)$, stage IV $\left(450-650^{\circ} \mathrm{C}\right)$, stage V $\left(650-1000^{\circ} \mathrm{C}\right)$ and stage VI $(1000$ $\left.1500^{\circ} \mathrm{C}\right)$. Stages III, IV and V have been closely investigated and are in accordance with the migration of monovacancies (III), bivacancies and impurity complexes (IV), and vacancy clusters (V) respectively. Self interstitials become mobile as low as $-170^{\circ} \mathrm{C}$, corresponding to stage I [7]. It is the purpose of this paper to correlate the annealing stages of tungsten with quantitative investigations of the annealed damage microstructures and provide plausible explanations for the evolving trends observed.

\section{EXPERIMENT AND RESULTS}

Pure tungsten (> 99.996 wt \%, $100 \mu \mathrm{m}$ sheet, Plansee Group) was heat treated in vacuum at $1400^{\circ} \mathrm{C}$ for 20 hours to remove dislocations introduced by processing. Discs of diameter $3 \mathrm{~mm}$ were punched and then electropolished to electron transparency using a twin-jet apparatus. A total of five R.T. irradiations with $150 \mathrm{keV} \mathrm{W}^{+}$ions were performed on the IVEMTandem at ANL to a fluence of $10^{18} \mathrm{~W}^{+} \mathrm{m}^{-2}(\sim 1.0 \mathrm{dpa})$ at a rate of $6.25 \times 10^{14} \mathrm{~W}^{+} \mathrm{m}^{-2} \mathrm{~s}^{-1}\left(\sim 5 \times 10^{-4}\right.$ 
$\left.\mathrm{dpa} \cdot \mathrm{s}^{-1}\right)$. Among which one was kept as a control sample, and the rest were then respectively annealed in-situ under the following conditions: $300^{\circ} \mathrm{C} / 120 \mathrm{~min}, 500^{\circ} \mathrm{C} / 120 \mathrm{~min}, 800^{\circ} \mathrm{C} / 30 \mathrm{~min}$ and $800^{\circ} \mathrm{C} / 120 \mathrm{~min}$. The rate of temperature ramp-up was $25^{\circ} \mathrm{C} / \mathrm{min}$. The uncertainty of temperature measurements originated mostly from the local heating of the $300 \mathrm{keV}$ electron beam. Fisher's model suggests an uncertainty of $5^{\circ} \mathrm{C}$ for tungsten [8].

The evolution of the damage microstructure was followed by analyses of dislocation loop number densities, spatial distributions, geometry and the interstitial/vacancy nature of loops. Figure 1 illustrates the time and temperature dependences of loop number densities. Here, the R.T. data presents the average loop populations found in all as-irradiated specimens. In addition, loop number densities directly correlate with the observations under weak-beam dark-field conditions of $\mathbf{g}= \pm 200 / 020$, 3-4g, i.e. they have not been corrected for contrast invisibility [9]. Within each run of annealing, a large reduction of the loop population took place during the ramp-up period before the full annealing temperature was achieved, as shown by the " 0 min" data, and the loop populations remained almost constant beyond $15 \mathrm{~min}$ of annealing. The final loop number density decreased with increasing annealing temperature, and was about $40 \%$ lower at $800^{\circ} \mathrm{C}$ than at $300^{\circ} \mathrm{C}$.

TEM micrographs of the damage microstructure are shown in Figure 2. At $300^{\circ} \mathrm{C}$ and $500^{\circ} \mathrm{C}$, the removal of $1-2 \mathrm{~nm}$ loops contributed most to the trend for decreasing number densities, whereas at $800^{\circ} \mathrm{C}$ loss of larger loops also contributed. At temperatures above stage III $\left(\geq 500^{\circ} \mathrm{C}\right)$ loop interactions and reactions gave rise to a transformation from a random distribution of loops to spatially ordered loops in the form of strings or rafts.

The geometry and nature of loops in tungsten as a function of annealing parameters are presented in Figures 3 and 4. Loops with Burgers vectors of both $1 / 2<111\rangle$ and $\langle 100\rangle$ types were found present, but the analysis of their absolute quantity as well as relative proportion was not performed based on a one-to-one correlation of loops. Instead, a statistical approach was applied, which involved the counting of loops for different $\mathbf{g}$ vectors and relied on the assumption that all variants of $1 / 2<111\rangle$ and $\langle 100\rangle$ were equal in quantity [10]. In Figure 3, it can be found that the increase of annealing temperature at above $300^{\circ} \mathrm{C}$ has resulted in the reduction of both $1 / 2<111\rangle$ and $\langle 100\rangle$ loops, but at a faster rate for $\langle 100\rangle$ loops (around a factor of 5 ). At $800^{\circ} \mathrm{C}$, over $80 \%$ of loops observed had $1 / 2<111>$ Burgers vectors. The increase of annealing time had similar but less pronounced effect on loop geometry.

The loop nature analyses in Figure 4 were based on inside-outside diffraction contrast behavior and include only $1 / 2<111>$ loops with diameter $\geq 4 \mathrm{~nm}$ [9]. Three major trends are shown. First, the fraction of interstitial loops increased with annealing temperature from around $35 \%$ at $300^{\circ} \mathrm{C}$ to around $50 \%$ at $\mathrm{T} \geq 500^{\circ} \mathrm{C}$. Second, the fraction of large $(>10 \mathrm{~nm})$ interstitial loops increased with annealing temperature. Third, large vacancy loops (>10 $\mathrm{nm}$ ) were also identified, but in smaller numbers. 


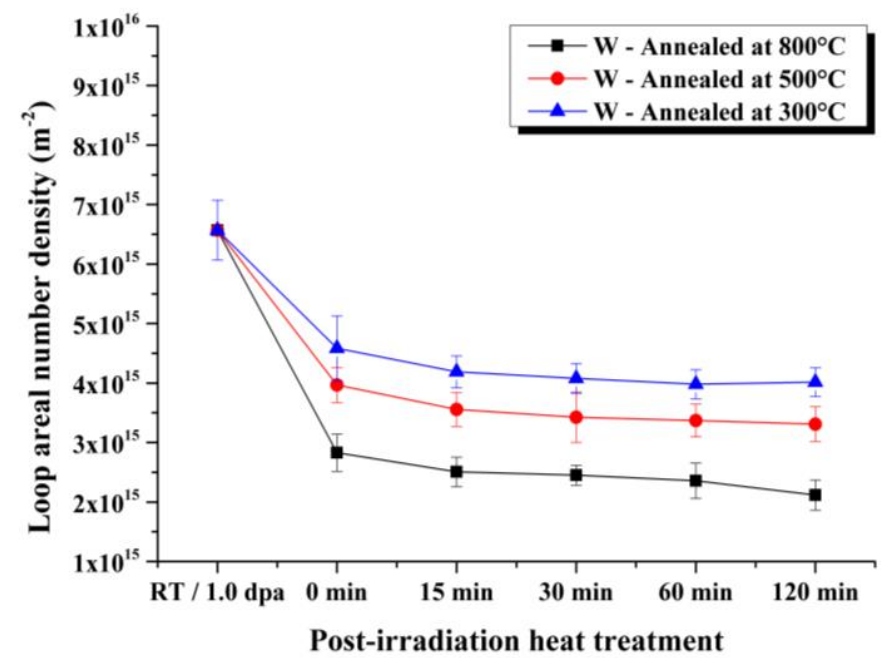

Figure 1. Evolution of loop number densities in tungsten (R.T. /1.0 dpa) as a function of annealing temperature and time.
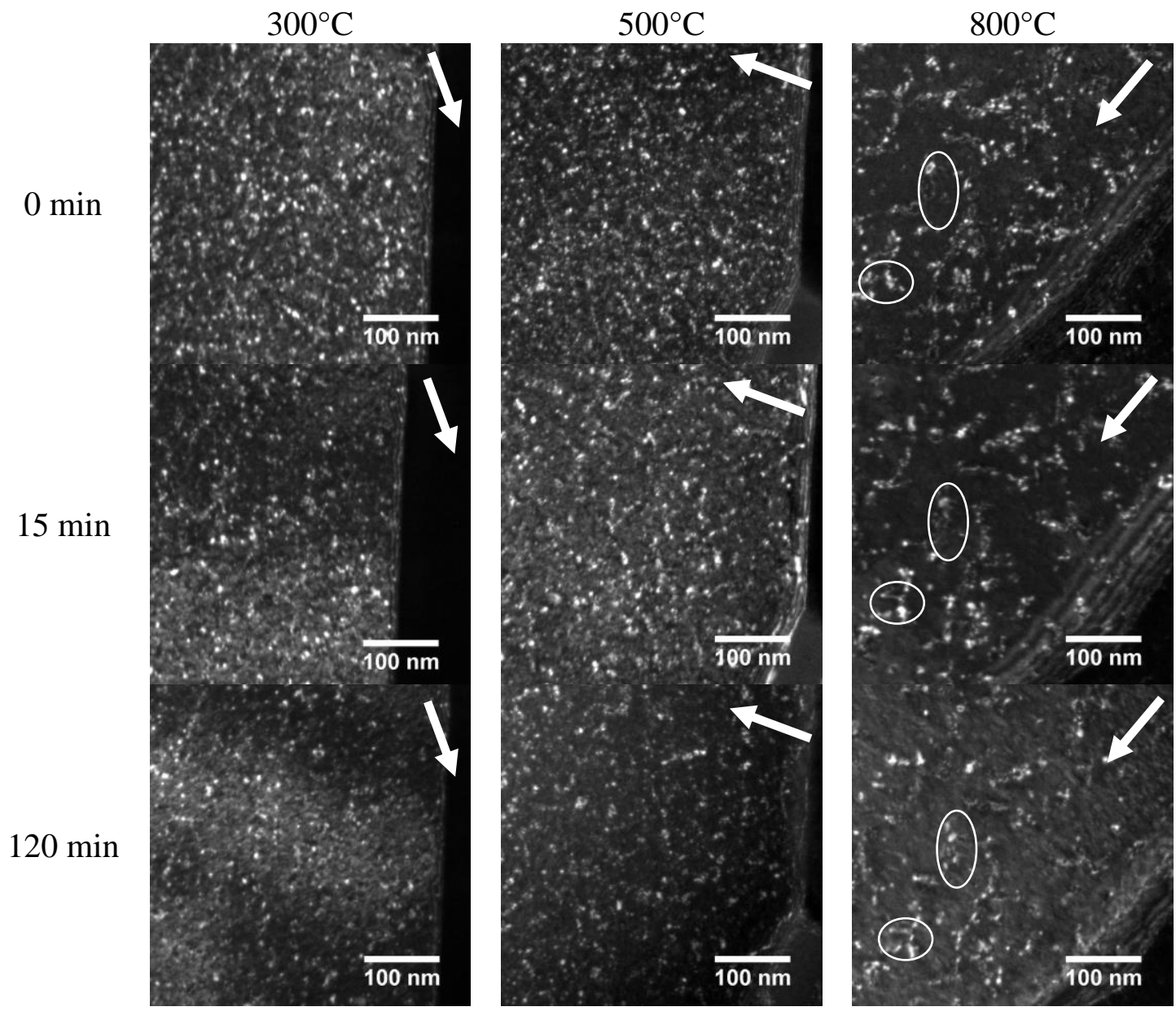

Figure 2. Microstructural evolution in tungsten (R.T. /1.0 dpa) as a function of annealing temperature and time. The thin-foils were imaged close to $\mathbf{z}=$ [001], in a weak-beam dark-field condition ( $\mathbf{g}=200,3-4 \mathbf{g}$, shown as the arrow). At $800^{\circ} \mathrm{C}$, loops which developed complex morphology are labelled with circles. 


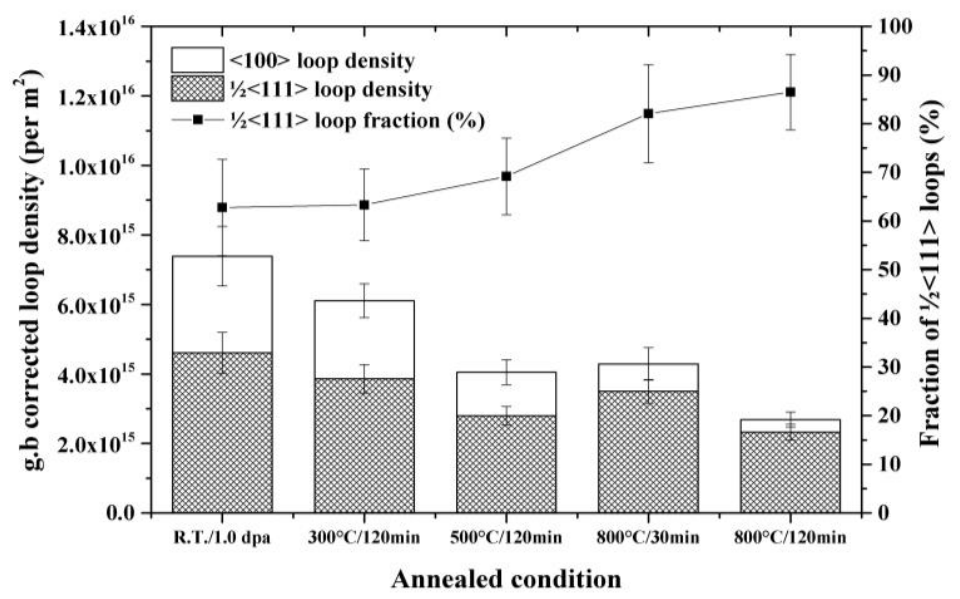

Figure 3. Invisibility corrected density and fraction of $1 / 2<111>$ loops in tungsten (R.T. /1.0 dpa) are presented as a function of annealing temperature and time.
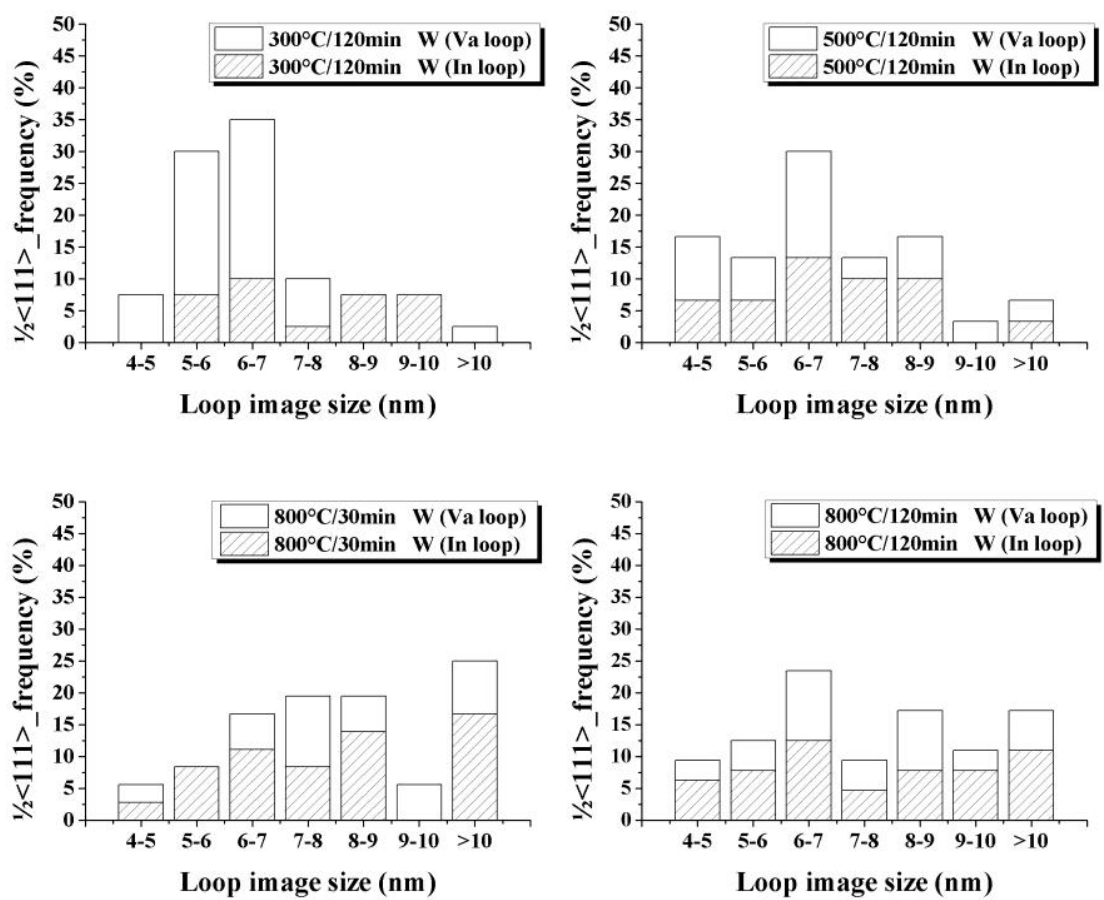

Figure 4. Nature and size analysis of $\mathbf{b}=1 / 2<111>$ loops in tungsten (R.T. /1.0 dpa) as a function of annealing temperature and time. The sampled bodies included typically 40-60 loops.

\section{DISCUSSION}

\section{Annealing stages versus microstructural evolution}

The evolution of damage microstructure during annealing is thought to proceed by migration and interaction of point defects (SIA, vacancies) and their corresponding clusters (loops, voids or small complexes formed with impurities) [11]. At $300^{\circ} \mathrm{C}$, corresponding to "stage III" of annealing, when only monovacancies are mobile, larger clusters of which would be stationary and serve as pinning objects during the glide motion of dislocation loops. The density 
of these pinning clusters were probably high. Although their dimensions were beyond the resolution limit of transmission electron microscopy, the hypothesis of a high density of pinning clusters in the damage microstructure can be verified from the observation that few loops exhibited long-range hopping behavior during the interval of annealing. Some loops changed in size, either growing or shrinking through the elastic attraction of point defects, adjacent clusters and loops, but the overall change in microstructure was minor.

At $500^{\circ} \mathrm{C}$, corresponding to "stage IV" of annealing, the mobility of monovacancies increases further and bivacancies start to become mobile as well. The transport of vacancies out of the original cascade was therefore effective, causing more interstitial loops to shrink and vacancy loops to grow. Also, the local barriers to loop migration were reduced, resulting in a growing number of absorption and coalescence reactions among loops. Consequently, loop number densities decreased and average loop sizes increased.

Judging from Figure 2, microstructural evolution during stages III and IV was mostly associated with the loss of 1-2 nm loops, and the reason for this is their higher diffusivity. An inverse proportional relationship between loop diameter and the diffusion coefficient is well established when loop migration follows the Arrhenius law [12], and such a trend has been verified experimentally in both iron [13] and tungsten [1].

Annealing at $800^{\circ} \mathrm{C}$, which corresponds to "stage V", is associated with the long-range migration of vacancy clusters, causing further loop growth or shrinkage, and leading to further loop detrapping. Loops became increasingly mobile and could migrate long distances along their glide cylinders. A particular outcome of this was the spatial ordering of the more mobile $1 / 2<111\rangle$ interstitial loops with the same Burgers vector variant, and their subsequent coalescence into finger-shaped loops when they were sufficiently close to each other. Many large loops also developed complex morphology during this stage (labelled with white circles in Figure 2). This might have been a result of the absorption of vacancies or vacancy clusters on their peripheries, leaving behind a number of small kinks.

DFT calculations show that the isotropic elasticity of tungsten prohibits the formation of $<100>$ loops. The $<111>$ crowdion is considered as the most stable geometry in tungsten and therefore $1 / 2<111>$ loops, which consist of bundles of $<111>$ crowdions are expected [11]. We have found that $\langle 100\rangle$ are unstable at elevated temperatures, consistent with theory, but why are such loops found at all and how did they vanish in the microstructure? Molecular dynamic simulations and low-dose irradiations on tungsten suggest that $<100>$ loops could originate as a quenched product of high energy collision cascade [14-15]. However, it is not yet clear what mechanism resulted in their loss. In addition to crowdions, DFT also predicted the relative stability of open and closed vacancy loops, of a range of dimensions. The most stable vacancy clusters under all circumstances were spherical voids [2]. The loss of vacancy loops may have been a consequence of their transformation to more stable $3 \mathrm{D}$ voids.

\section{Effects of the foil surface on interpretations of annealing behavior}

The influence of the foil surface should be considered in thin foil experiments. According to SRIM, the displacement damage introduced by $150 \mathrm{keV} \mathrm{W}^{+}$ions is produced within $30 \mathrm{~nm}$ of the surface [15]. Dislocation loops, in particular $1 / 2<111>$ loops, are prone to loss to the surface through glide. $<100>$ loops are less mobile and the loss of such loops is expected to be negligible, although the loss of [100] loops to the surface has been seen in [001] foils of Fe [16]. In general, an underestimation of loop number densities, the fraction of $1 / 2<111>$ loops and of 
large interstitial loops is likely. However, the characteristic annealing behavior identified, i.e. the temperature and time at which $\langle 100\rangle$ loops lose thermal stability and the transition points for damage microstructural changes should not be affected. Bulk annealing experiments have been carried out on the same batch of material, but irradiated with $2 \mathrm{MeV} \mathrm{W}{ }^{+}$ions to the same fluence [17]. The results not only confirm the observations in this work, but also provide quantitative insights of the material performance in bulk, where the surface attraction was minimized.

\section{CONCLUSIONS}

In-situ annealing was performed to investigate the thermal stability of radiation damage in tungsten. Temperature played the dominant role in affecting loop number densities, geometry, and size-nature distributions. The evolution of damage microstructure was less sensitive to time.

\section{ACKNOWLEDGMENTS}

The in-situ irradiation and annealing experiments were accomplished at the Electron Microscopy Center for Materials Research at Argonne National Laboratory, a US Department of Energy Office of Science Laboratory operated under Contract No. DE-AC02-06CH11357 by UChicago Argonne, LLC. We thank Mr. Pete Baldo for his help with the irradiations. Many thanks to EPSRC (EP/H018921/1) and the EFDA mobility scheme for funding this research.

\section{REFERENCES}

[1] X. Yi, PhD. Thesis, University of Oxford, 2013.

[2] M.R. Gilbert, S.L. Dudarev, P.M. Derlet and D.G. Pettifor, J. Phys.: Condens. Matter. 20, 345214 (2008).

[3] M. Attardo and J.M. Galligan, Phys. Stat. Sol. 16, 449 (1966).

[4] K.M. Bowkett and B. Ralph, Proc. Roy. Soc. A. 312, 51-63 (1969).

[5] D. Jeannotte and J.M. Galligan, Acta Metallurgica. 18, 71-79 (1970).

[6] L.K. Keys, J.P. Smith and J. Moteff, Phys. Rev. 176 (3), 851-856 (1968).

[7] V.N. Bykov, G.A. Birzhevoi, M.I. Zakharova, V.A. Solov'ev, Atomic Energy. 33 (4), 930-

935 (1972) [Soviet Atomnaya Énergiya. 33 (4), 809-813 (1972)]

[8] S.B. Fisher, Radiation Effects. 5, 239-243 (1970).

[9] M.L. Jenkins and M.A. Kirk, Characterization of Radiation Damage by Transmission Electron Microscopy. (Institute of Physics Publishing, Bristol and Philadelphia, 2001) p. 31, 74. [10] A. Prokhodtseva, B. Décamps, A. Ramar and R. Schäublin, Acta Materialia. 61 (18), 69586971 (2013).

[11] S.L. Dudarev, Annu. Rev. Mater. Res. 43, 35-61 (2013).

[12] H. Mehrer, Diffusion in Solids. (Springer-Verlag, Berlin and Heidelberg, 2007) p. 127.

[13] K. Arakawa, K. Ono, M. Isshiki, K. Mimura, M. Uchikoshi and H. Mori, Science. 318, 956959 (2007).

[14] A.E. Sand, S.L. Dudarev and K. Nordlund, arXiv:1306.3824 [cond-mat.mtrl-sci].

[15] D.R. Mason, X. Yi, M.A.Kirk and S.L. Dudarev, arXiv:1402.0689 [cond-mat.mtrl-sci].

[16] M.L. Jenkins, Z. Yao, M. Hernández-Mayoral, M.A. Kirk, J. Nucl. Mater. 389, 197-202 (2009).

[17] F. Ferroni, X. Yi, P. Edmondson and S.G. Roberts (private communication). 